\title{
Atrial fibrosis in a chronic murine model of obstructive sleep apnea: mechanisms and prevention by mesenchymal stem cells
}

Pablo Ramos ${ }^{1}$, Cira Rubies ${ }^{1}$, Marta Torres ${ }^{2}$, Montserrat Batlle ${ }^{1}$, Ramon Farre ${ }^{3}$, Josep Brugada ${ }^{1}$, Josep M Montserrat ${ }^{2}$, Isaac Almendros ${ }^{2+}$ and Lluís Mont ${ }^{1 * \dagger}$

\begin{abstract}
Background: OSA increases atrial fibrillation (AF) risk and is associated with poor AF treatment outcomes. However, a causal association is not firmly established and the mechanisms involved are poorly understood. The aims of this work were to determine whether chronic obstructive sleep apnea (OSA) induces an atrial pro-arrhythmogenic substrate and to explore whether mesenchymal stem cells (MSC) are able to prevent it in a rat model of OSA.

Methods: A custom-made setup was used to mimic recurrent OSA-like airway obstructions in rats. OSA-rats $(n=16)$ were subjected to 15 -second obstructions, 60 apneas/hour, 6 hours/day during 21 consecutive days. Sham rats $(n=14)$ were placed in the setup but no obstructions were applied. In a second series of rats, MSC were administered to OSA-rats and saline to Sham-rats. Myocardial collagen deposit was evaluated in Picrosirius-red stained samples. mRNA expression of genes involved in collagen turnover, inflammation and oxidative stress were quantified by real time PCR. MMP-2 protein levels were quantified by Western Blot.

Results: A 43\% greater interstitial collagen fraction was observed in the atria, but not in the ventricles, of OSA-rats compared to Sham-rats (Sham $8.32 \pm 0.46 \%$ vs OSA $11.90 \pm 0.59 \%, \mathrm{P}<0.01$ ). Angiotensin-I Converting Enzyme (ACE) and Interleukin 6 (IL-6) expression were significantly increased in both atria, while Matrix Metalloproteinase-2 (MMP-2) expression was decreased. MSC administration blunted OSA-induced atrial fibrosis (Sham + Saline $8.39 \pm$ $0.56 \%$ vs OSA + MSC $9.57 \pm 0.31 \%, P=0.11$ ), as well as changes in MMP-2 and IL-6 expression. Interleukin 1- $\beta$ (IL-1 $\beta$ ) plasma concentration correlated to atrial but not ventricular fibrosis. Notably, a 2.5-fold increase in IL-1 $\beta$ plasma levels was observed in the OSA group, which was prevented in rats receiving MSC.
\end{abstract}

Conclusions: OSA induces selective atrial fibrosis in a chronic murine model, which can be mediated in part by the systemic and local inflammation and by decreased collagen-degradation. MSCs transplantation prevents atrial fibrosis, suggesting that these stem cells could counterbalance inflammation in OSA.

Keywords: Obstructive sleep apnea, Atrial fibrillation, Cardiac fibrosis, Mesenchymal stem cells, Animal model

\footnotetext{
* Correspondence: Imont@clinic.ub.es

${ }^{\dagger}$ Equal contributors

'Thorax Institute, Unitat de Fibril · lació Auricular, Hospital Clínic. Universitat de Barcelona and Institut d'Investigacions Biomèdiques August Pi i Sunyer (IDIBAPS), Barcelona, Catalonia, Spain

Full list of author information is available at the end of the article
}

\section{Biomed Central}

(c) 2014 Ramos et al.; licensee BioMed Central Ltd. This is an Open Access article distributed under the terms of the Creative Commons Attribution License (http://creativecommons.org/licenses/by/2.0), which permits unrestricted use, distribution, and reproduction in any medium, provided the original work is properly credited. The Creative Commons Public Domain Dedication waiver (http://creativecommons.org/publicdomain/zero/1.0/) applies to the data made available in this article, unless otherwise stated. 


\section{Background}

Patients with obstructive sleep apnea (OSA) show both a high prevalence [1] and incidence [2] of atrial fibrillation (AF). In addition, OSA has been associated with a greater risk of AF recurrence after cardioversion [3] and catheter ablation $[4,5]$ and a worse response to antiarrhythmic drugs [6]. Despite the clear association between OSA and AF, it is not firmly established whether this association is causal or mediated by other comorbidities often present in OSA-patients, such as obesity or hypertension [7].

Atrial structural remodeling, particularly fibrosis, is a main component in the substrate predisposing to AF [8]. Atrial fibrosis predicts disease progression and treatment outcomes [9]. It is known from murine models that exposure to recurrent airway obstructions promotes early myocardial inflammation leading to myocardial apoptosis at mid-term [10]. However, it remains unknown whether chronic exposure to recurrent apneas can reach to develop atrial fibrosis, thus explaining the higher prevalence and incidence of AF observed in OSA patients. In addition, cell-based therapies emerge as an attractive alternative to classic pharmacological treatments for the prevention of such remodeling, thereby reducing AF occurrence and progression. Among the options available for cell therapy, bone marrow mesenchymal stem cells (MSC) appear as a promising source of stem cells because of their multi-lineage potential, antiinflammatory effects $[11,12]$, ability to escape detection by the host immune system, and a relative ease of expansion in culture $[13,14]$. Recent studies have shown that MSCs attenuate cardiac fibrosis in a variety of experimental settings [15-17]. Although the knowledge on the therapeutic role of MSC in OSA models is very limited [18], there is evidence that stem cells possess anti-inflammatory properties that mitigate the early inflammatory response [11].

The aim of our study was 1) to describe OSA-induced atrial remodeling in a chronic murine model, 2) to analyze the putative mechanisms involved and 3) to investigate whether MSC have the potential to prevent such remodeling in the same OSA model.

\section{Methods}

\section{Experimental sleep apnea model}

This study conformed to European Community (Directive 86/609/EEC) and Spanish guidelines for the use of experimental animals and was approved by the Animal Research Ethics Committee of the University of Barcelona.

A chronic model of OSA previously validated by our group was used [19]. The model was designed to apply recurrent airway obstructions with an OSA pattern. Briefly, it was based on a custom-made setup consisting of 2 chambers (to fit the body and head) separated by a latex neck collar (Figure 1). The head chamber had a conical shape and was built small enough to contain the minimum possible air volume when the rat was in place. The rat breathed room air through an orifice at the vertex of the conical head chamber. A valve was placed at the entrance of the head chamber, allowing for the closure of the orifice. The valve was electronically controlled to produce intermittent obstructions mimicking those that characterize OSA, resulting in increased breathing efforts, oxygen desaturations and intermittent hypercapnia. To ensure the development of obstructive apneas, the head chamber was connected to air flow, pressure and $\mathrm{CO}_{2}$ transducers; pulse oximetry was measured at the rat tail. These parameters were continuously monitored by an experimented researcher who certified effective apneas (i.e., no air flow, pressure swings, increased $\mathrm{CO}_{2}$ and decreased pulse oximetry).

The first part of the study was carried out in 30 SpragueDawley male rats (250-300 g and 8 weeks old at the beginning of the experiment) randomized into 2 groups: OSA and Sham. OSA-rats $(n=16)$ were subjected to 15 second obstructions at a rate of 60 per hour, 6 hours per day during 21 consecutive days. Sham-rats $(n=14)$ were placed in the setup during the same period of time (21 days, 6 hours per day), but no obstructions were applied. Rats in both groups were progressively adapted to the experimental setting by increasing the time within the OSA/Sham device at a rate of 1 hour each day, up to $6 \mathrm{~h}$ at the end of the first week. Rats were housed in a controlled environment (12/12-hour light/dark cycle) and fed rodent chow and tap water ad libitum.

\section{Mesenchymal stem cells infusion}

To evaluate the effect of MSC in OSA-induced structural remodeling, 8 rats undergoing the previously described OSA protocol were infused with MSC (OSA + MSC group). Seven Sham rats receiving saline vehicle (Sham + $S$ group) were used as controls in this experiment.

MSC were obtained by culturing well-characterized Lewis rat marrow stromal cells kindly provided by the

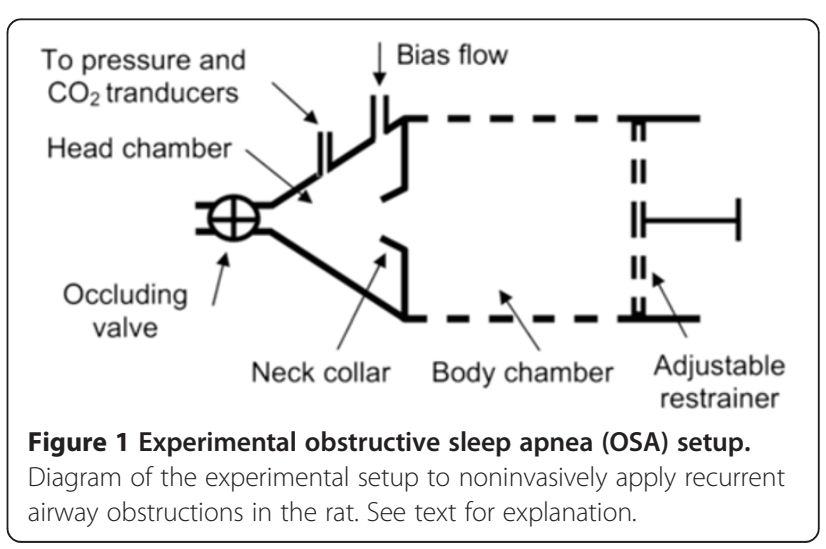


Tulane Center for Gene Therapy (New Orleans, LA, USA). The cells were cultured in MEM-alpha medium with glutamine and without ribonucleosides or deoxyribonucleosides (GIBCO, Gaithersburg, MD, USA), supplemented with $20 \%$ fetal bovine serum (HyClone Cell Culture, Cultek, Madrid, Spain), 1\% antibiotic-antimycotic (containing 10,000 U/mL Penicillin G sodium; 10,000 $\mu \mathrm{g} / \mathrm{mL}$ Streptomycin sulphate; $25 \mu \mathrm{g} / \mathrm{mL}$ Amphotericin B as Fungizone [GIBCO, Gaithersburg, MD, USA]) and 2\% l-glutamine (GIBCO, Gaithersburg, MD, USA). The cells were grown at $37^{\circ} \mathrm{C}, 5 \% \mathrm{CO} 2,100 \%$ humidity. Subconfluent cells were dissociated with $0.25 \%$ trypsin and $1 \mathrm{mM}$ Ethylene Diamine Tetraacetic Acid (EDTA) in Hanks' Balanced Salt Solution (GIBCO, Gaithersburg, MD, USA) and subcultured at low density in new culture flasks. To prepare the injection, MSC were trypsinized and $5 \times 10^{6}$ cells were suspended in $500 \mu \mathrm{L}$ of Phosphate Buffered Saline (GIBCO, Gaithersburg, MD, USA). This cell preparation was slowly delivered (30 seconds) to the rat through the penile vein the first day of apneas application and every 4 days thereafter. Rats were anaesthetized with short-acting inhaled isoflourane (2\%) before and during every injection.

All rats in the four experimental groups (Sham, Sham $+S$, OSA, OSA + MSC) were carefully inspected daily to monitor animal well-being. No rats showed any signs of stress or MSC-related side effects and thus, no rats needed to be excluded from the analysis.

\section{Sample collection and RNA isolation}

Once anesthetized with intraperitoneal urethane 10\% ( $1 \mathrm{~g} / \mathrm{kg})$, animals were sacrificed by exsanguination through carotid artery cannulation. Blood samples were collected in EDTA tubes and centrifuged for 15 minutes at $3000 \mathrm{rpm}$. Plasma was collected and frozen at $-80^{\circ} \mathrm{C}$.

Hearts were quickly removed and weighted, and tissue samples were obtained from the left ventricle (LV) free wall, right ventricle (RV) free wall, and both atrial appendages: right atrium (RA) and left atrium (LA). Samples were snap-frozen in liquid nitrogen for posterior molecular biology analysis. For histological studies, transversal sections from the midventricular and basal (including both atria) regions of the heart were obtained, fixed in formol $3 \%$ for 24 hours and embedded in paraffin.

Total RNA was isolated from the 4 cardiac chambers. Myocardial tissue was first homogenized with Trizol $^{\odot}$ reagent (Life Technologies) and further purified with chloroform (C-2432, Sigma-Aldrich). Final RNA was obtained with silica columns (RNA Aqueous kit, Ambion) according to the manufacturer's protocol. The integrity of the resulting RNA was assessed in formaldehydedenaturing agarose gels. A reverse transcription protocol with random primers was applied to $1 \mu \mathrm{g}$ of total RNA for cDNA synthesis with the addition of RNAse inhibitors (High capacity cDNA RT kit, Life Technologies, CA, USA) with a MJ Research PTC 200 thermal cycler (MJ Research, MA, USA).

\section{Real time polymerase chain reaction (real time PCR)}

Messenger RNA (mRNA) expression of selected keyplayer genes in the collagen synthesis and degradation balance was measured in the four cardiac chambers. Genes involved in collagen-synthesis promotion (ACE, TGF- $\beta 1$ ), collagen maturation and cross-linking (LOX), and degradation (MMP-2, MMP-3, MMP-9, MMP-10, TIMP-1, TIMP-2), as well as oxidative stress (eNOS) and inflammation (IL-6) were quantified. mRNA expression was assayed with a real-time PCR 7900 thermal cycler (AB, Applied Biosystems, CA, USA) using TaqMan Universal PCR Master Mix with AmpErase UNG. Specific TaqMan single-gene expression assays are shown in Table 1. All mRNA quantification results are shown relative to a cDNA pool with sequential dilutions and units are given as ng-equivalents of cDNA (ng-equ).

\section{Protein isolation and analysis by western blot}

Myocardial proteins were extracted from each heart cavity in all experimental groups. Samples were submerged in $0.5 \mathrm{~mL}$ of ice-cold protein lysis buffer containing: RIPA buffer (R0278, Sigma), $1 \mathrm{mM}$ phenylmethanesulfonyl fluoride (P7626, Sigma), $1 \mathrm{mM}$ sodium orthovanadate (S6508, Sigma), 1 mM Pefabloc (11429868001, Roche) and complete Mini Protease Inhibitor Cocktail (11836153001, Roche). Samples were homogenized with an Omni TH homogenizer (Omni International Inc.). After 1 hour of rotation at $4^{\circ} \mathrm{C}$, samples were centrifuged at $10.000 \mathrm{~g}$ at $4^{\circ} \mathrm{C}$ for 30 minutes. The upper phase was collected and the total protein concentration was quantified with the Pierce BCA protein Assay method (23227, Thermo Scientific, Pierce) relative to a BSA standard curve. Forty

\section{Table 1 TaqMan one-gene expression assays}

\begin{tabular}{lll}
\hline Gene & Abbreviation & $\begin{array}{l}\text { TaqMan } \\
\text { reference }\end{array}$ \\
\hline Angiotensin-I Converting Enzyme & ACE1 & Rn00561094_m1 \\
Transforming Growth Factor- $\beta 1$ & TGF- 31 & Rn00572010_m1 \\
Lysyl Oxidase & LOX & Rn01491829_m1 \\
Matrix Metallopeptidase-2 & MMP-2 & Rn01538170_m1 \\
Matrix Metallopeptidase-3 & MMP-3 & Rn00591740_m1 \\
Matrix Metallopeptidase-9 & MMP-9 & Rn00579162_m1 \\
Matrix Metallopeptidase-10 & MMP-10 & Rn00591678_m1 \\
Tissue Inhibitor of Metallopeptidase-1 & TIMP1 & Rn00587558-m1 \\
Tissue Inhibitor of Metallopeptidase-2 & TIMP2 & Rn00573232_m1 \\
Nitric Oxide Synthase III & NOS3 & Rn02132634_s1 \\
Interleukin-6 & IL-6 & Rn01410330_m1 \\
\hline
\end{tabular}


$\mu \mathrm{g}$ of total protein extract were loaded with a reducing buffer $(2.5 \%$ of $\beta$-mercaptoethanol) to NuPage $4-12 \%$ Bis-Tris Gel (NP0322). A western blot was performed with the Novex ${ }^{\oplus}$ gels methodology (Invitrogen). Proteins were transferred to a nitrocellulose membrane using a blot gel transfer (IB3010-01) and the iBlot ${ }^{\circ}$ Dry Blotting System. Proper transfer was checked by Ponceau staining. After 1 hour blockade of the membrane with phosphate buffered saline solution (PBS, Fisher Scientific), 0.1\% Tween 20 (P1379, Sigma-Aldrich) and 5\% of skimmed milk, it was incubated overnight at $4^{\circ} \mathrm{C}$ with the MMP-2 primary antibody (ab37150, Abcam) diluted 1/500. Afterwards, the membrane was incubated during 1 hour with an HRPGoat anti rabbit secondary antibody diluted $1 / 1000$ (31460 Thermo Scientific). Final detection of the MMP-2 protein bands was accomplished with the ECL kit Supersignal West Pico Chemioluminescent Substrate (34080, Thermo Scientific).

Bands around $60 \mathrm{KDa}$ for MMP-2 (in antibody and ECL incubated membranes) and around $40 \mathrm{KDa}$ (in Ponceau-stained membranes) were quantified (ImageJ, NIH, Maryland, USA). Loading of each sample was normalized with the Ponceau band around $40 \mathrm{KDa}$. Results are given in arbitrary units (A.U.) as the ratio between the normalized densities of each sample divided by the normalized density of a standard loaded in each gel.

\section{Plasma cytokines}

Systemic inflammation-related cytokines -proinflammatory and profibrotic Interleukin-1 beta (IL-1 $\beta$ ), and anti-inflammatory Interleukin-10 (IL-10)- were measured in plasma obtained at sacrifice with Quantikine ELISA assays RLB00 and R1000, respectively (R\&D Systems, MN, USA).

\section{CD90 immunofluorescence}

A CD90 immunofluorescence assay was performed in myocardial parafine-embedded sections from rats injected with MSC, and in MSC cultured in 30-40\% confluent chamber slides (positive control). Cultured MSC were fixed with $4 \%$ paraformaldehyde. Antigenic retrieval was achieved with a sodium citrate $10 \mathrm{mM}$ bath at $80^{\circ} \mathrm{C}$ during $40 \mathrm{~min}$. After blockade of unspecific unions, samples were incubated overnight with Anti-Rat CD90, FITC conjugated antibody (MR5001, Caltag, Invitrogen) diluted $1 / 200$. Sudan Black was used to mask auto-fluorescence. Both myocardial sections and chambers were added a DAPI-containing mounting media and covered. The same staining protocol excluding Anti-CD90 antibody addition was used as a negative control for both groups.

\section{Myocardial fibrosis quantification}

Mid-ventricular and atrial sections of paraffin-embedded tissue, 4 microns thick, were stained with Picrosirius-red as previously described [20]. Four random pictures $(40 \times)$ from RV and LV free wall, and 6 random pictures $(100 \times)$ from the atria were taken from each sample with an Olympus BX51 microscope and an Olympus DP50 camera (Olympus Corporation, Japan).

To estimate hypertrophy of the cardiac chambers, RV free wall, interventricular septum (IVS) and LV free wall thickness were measured in transversal sections at midventricular level. The interstitial collagen fraction was assessed with a semiautomatic color-threshold detection software (AnalySIS ${ }^{\oplus}$, Soft Imaging System, Germany). Right and left atrial collagen fraction were quantified together. Epicardial, endocardial, and perivascular fibrosis were excluded from the analysis. All measures were carried out blind by a single investigator.

\section{Statistical analysis}

All variables followed a normal distribution (ShapiroWilks test) and are expressed as mean \pm standard error of the mean (SEM). Grubbs test was used to exclude extreme outliers (maximum one per group). Comparisons between 2 groups were carried out with a non-paired Student's t-test. A Pearson product-moment correlation coefficient was computed to assess the relationship between the plasmatic IL-1 $\beta$ levels and the myocardial collagen fraction. Statistical calculations were performed with SPSS v16.0 (SPSS Institute Inc, Cary, NC, USA). A $\mathrm{p}$-value $<0.05$ was considered for significance.

\section{Results}

\section{Obstructive sleep apnea induces atrial fibrosis Histological analysis}

The 3-week OSA protocol did not induce global or specific-chamber hypertrophy in rats. No significant differences between both groups were found in heart weight (Sham $1.21 \pm 0.02$ g. vs OSA $1.14 \pm 0.03$ g, $\mathrm{P}=0.08$ ), RV free wall thickness (Sham $938 \pm 55 \mu \mathrm{m}$ vs OSA $876 \pm$ $65 \mu \mathrm{m}, \mathrm{P}=0.49$ ), IVS thickness (Sham $2234 \pm 162 \mu \mathrm{m}$ vs OSA $2069 \pm 190 \mu \mathrm{m}, \mathrm{P}=0.54$ ) or LV free wall thickness (Sham $2427 \pm 178 \mu \mathrm{m}$ vs OSA $2300 \pm 190 \mu \mathrm{m}, \mathrm{P}=0.64$ ).

We evaluated myocardial fibrosis in histological preparations. Figure 2A shows representative photomicrographs of Picrosirius-red stained LV, RV and atrial sections from Sham and OSA rats. OSA induced a significant $43 \%$ higher atrial interstitial collagen deposition as compared to the sham group (Figure 2B). Increased fibrosis deposition was diffuse and homogenous, with no patchy fibrotic infiltrates in any of the analyzed samples. No differences in collagen deposit density were observed between OSA and Sham groups in RV and LV free wall.

\section{mRNA expression and protein synthesis}

To study the mechanisms of myocardial fibrosis, we quantified mRNA expression of genes involved in myocardial collagen turnover, inflammation and oxidative 


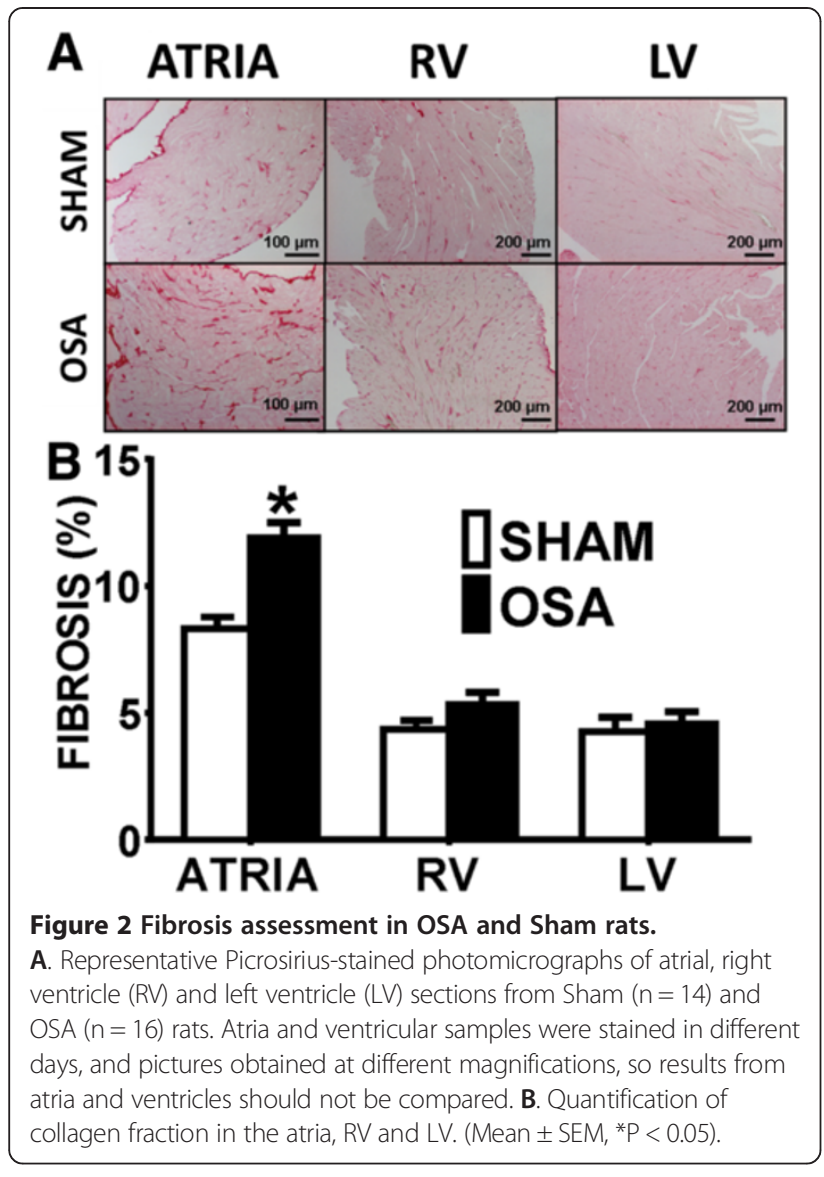

stress in the 4 cardiac chambers of sham and OSA-rats. ACE expression was significantly greater in the RA, RV and LV, and was close to significance $(p=0.055)$ in the LA, of OSA-rats compared with Sham-rats (Figure 3A). Expression of collagen promoters TGF- $\beta 1$ and LOX was unaltered (Figure 4D, F). Regarding collagen degradation, MMP-2 expression was significantly decreased in both atria, but not in the ventricles (Figure 3B). No significant changes were found in any other metalloproteinase or metalloproteinase inhibitor (Figure 4A-C). MMP-3 and MMP-10 levels were undetectable and are not shown. Finally, IL-6 expression was significantly higher in both atria and in the LV of the OSA group, but showed no differences in the RV (Figure 3C).

As a potential regulator of myocardial fibrosis in our model, protein levels of MMP-2 were analyzed in the four cardiac chambers. Representative blots and quantification are shown in Figure 3E. MMP-2 protein levels were lower in left atria from OSA-rats than in left atria from Sham-rats $(p=0.03)$, and showed borderline significance for the right atrium $(p=0.06)$. No differences were found between Sham and OSA groups in the left and right ventricles.

\section{Plasma cytokines}

OSA-induced changes in systemic inflammation were studied in plasma samples. A remarkable 2.5-fold increase in pro-inflammatory IL-1 $\beta$ plasma levels was observed in the OSA group compared to the sham group (Figure 3D). IL-1 $\beta$ plasma levels positively correlated to atrial $(r=0.404$, $\mathrm{p}=0.037$, Figure $5 \mathrm{~A})$, but not ventricular $(\mathrm{p}=0.21$, Figure 5B) collagen fraction. Conversely, no differences were found in IL-10 plasma levels between both groups (Sham $11.25 \pm 2.96 \mathrm{pg} / \mathrm{mL}$ vs OSA $7.85 \pm 1.41 \mathrm{pg} / \mathrm{mL}$, $\mathrm{P}=0.26$ ). Plasma IL-10 did not correlate to either atrial or ventricular collagen fraction (results not shown, $\mathrm{p}=0.209$ and $\mathrm{p}=0.373$, respectively).

\section{Mesenchymal stem cells prevent sleep apnea-induced atrial fibrosis Histological analysis}

As in the first set of rats, no differences between OSA + MSC and Sham $+\mathrm{S}$ groups were found in heart weight nor in the ventricular collagen fraction. OSA-induced atrial fibrosis was prevented by the administration of MSC; OSA + MSC and Sham + S groups showed a similar atrial collagen fraction (Figure 6).

\section{CD90 immunofluorescence}

We used the sensitive MSC-marker CD90 to study their presence in the myocardium of OSA + MSC rats. Figure 7 shows representative microphotographies of a positive control (cultured MSC in A-B) and sample of interest (LA from OSA + MSC rats in C-D). After thoroughly exploring the myocardium samples, no CD90 positive cells were found in the atria or ventricles of five OSA + MSC rats myocardial tissue-sections.

\section{mRNA expression and protein synthesis}

Beneficial effects of MSC infusion in the atria of OSA rats were not accompanied by regression of changes in ACE mRNA expression. ACE mRNA expression remained higher in the RA and LA of OSA + MSC rats compared with Sham $+S$ rats (Figure 8A). Conversely, MSC blunted OSA-induced atrial MMP-2 downregulation; MMP-2 expression was similar in OSA + MSC and Sham $+S$ groups (Figure 8B). IL-6 OSA-induced increase was reverted after MSC-infusion (Figure 8C). No differences were observed in the expression of the other genes analyzed in the atria, though changes of uncertain significance were found for MMP-2 (Figure 8B), eNOS and TGF- $\beta 1$ in the LV (Figure 9).

Results for MMP-2 mRNA expression in the atria were confirmed in protein levels quantification (Figure 8E). No differences were found in MMP-2 protein levels between Sham $+\mathrm{S}$ and OSA + MSC rats in the left or right atria. Contrary to mRNA expression, no differences were 


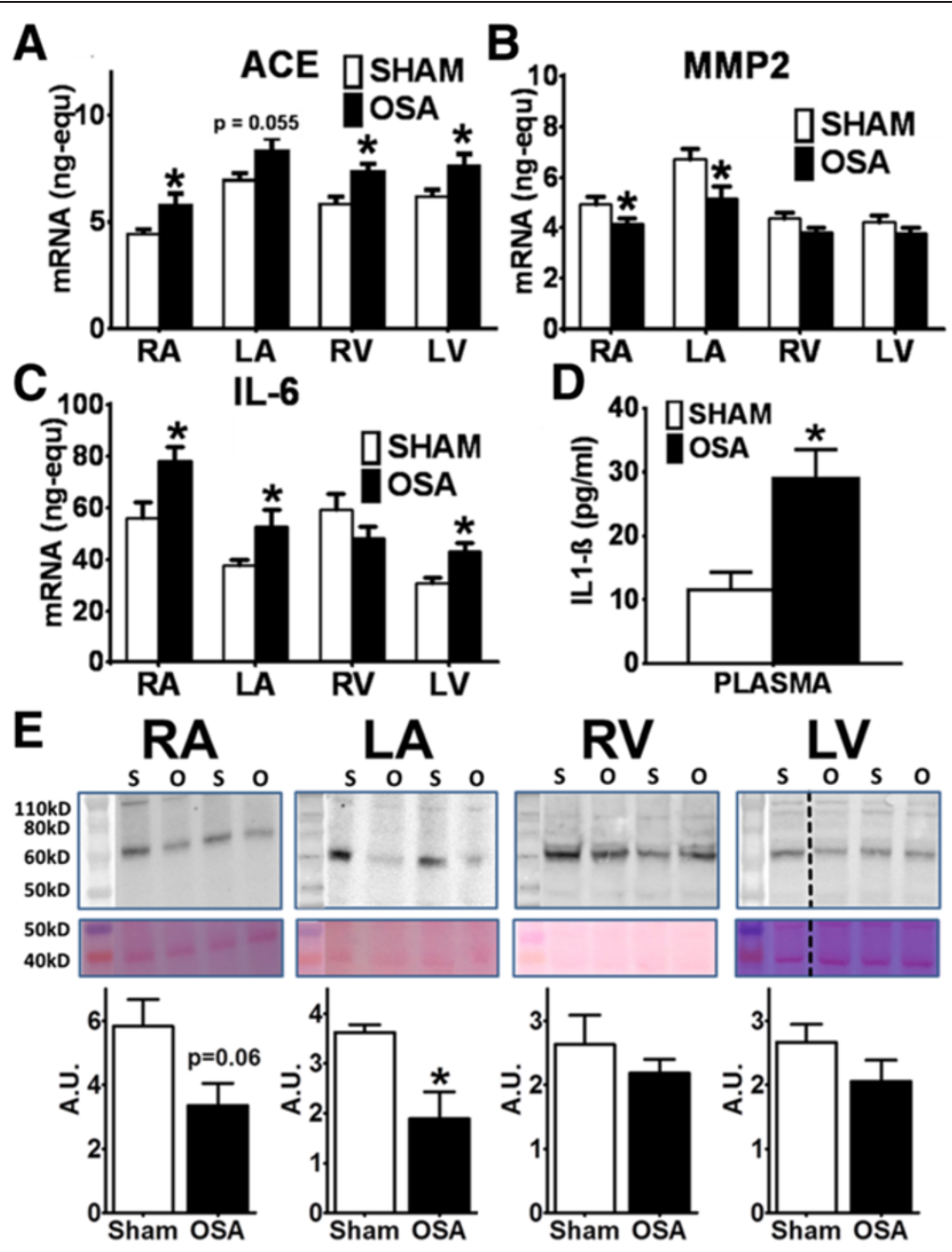

Figure 3 Fibrosis mechanisms assessment in OSA and Sham rats. (A) ACE, (B) MMP-2 and (C) IL-6 mRNA expression (ng-equ) in the four cardiac chambers of Sham $(n=14)$ and OSA $(n=16)$ rats (Mean $\pm S E M$, $\left.{ }^{* P}<0.05\right)$. D. Plasmatic concentration $(p g / m L)$ of IL-1 in Sham and OSA (Mean \pm SEM, ${ }^{*} \mathrm{P}<0.05$ ). E. MMP-2 protein levels quantification. Representative MMP-2 blots (upper panel), Ponceau-stained membranes (middle panel) and normalized quantification (lower panel) (Mean \pm SEM, $\left.{ }^{*} \mathrm{P}<0.05\right)$. $n=4-6 /$ group. Left lane is a molecular-weight marker lane; the picture was obtained with visible light. Dashed line means non-contiguous lane. A.U.: Arbitrary Units.

seen in MMP-2 protein levels in the $\mathrm{LV}(\mathrm{Sham}+\mathrm{S}$ vs OSA + MSC).

\section{Plasma cytokines}

Figure $8 \mathrm{D}$ shows mean IL- $1 \beta$ levels in the Sham $+\mathrm{S}$ and OSA + MSC groups. OSA-induced increase in IL-1 $\beta$ plasma levels was blunted in the OSA group receiving MSC.

\section{Discussion}

In the present work, we have demonstrated for the first time that chronic exposure of rats to recurrent apneas can promote atrial fibrosis, an established substrate for AF. This process seems to be mainly mediated through an increased local and systemic inflammation and a reduced collagen-degradation. Also, our data revealed that the OSA-induced fibrosis can be prevented by the infusion of MSC.

The application of intermittent hypoxia by modifying oxygen concentration in breathed gas, which is the most widely used model in OSA, is able to induce systemic inflammation. However, the setting employed in our work, in addition to intermittent hypoxia, allowed the application of increased negative intrathoracic-pressure swings [19]. These increased inspiratory efforts could aggravate the early local inflammatory response triggered by intermittent hypoxia alone. In addition, both factors could participate in the development of AF by different mechanisms; i.e. intermittent hypoxia can elevate the systemic blood pressure but also, increased intrathoracic pressure swings can increase left ventricular transmural pressure [21] and may lead to repetitive atrial stretch 


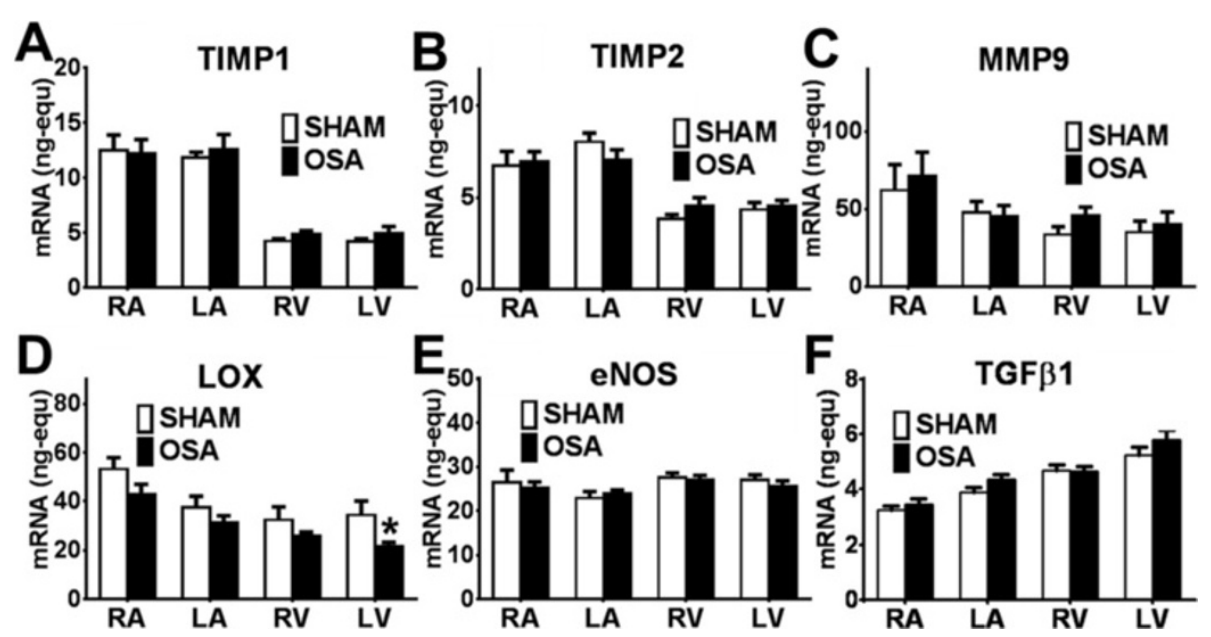

Figure 4 mRNA expression in the OSA and Sham rats. (A) TIMP-1, (B) TIMP-2, (C) MMP-9, (D) LOX, (E) eNOS and (F) TGF- $\beta 1$ mRNA expression (ng-equ) in the four cardiac chambers of Sham $(n=14)$ and OSA $(n=16)$ rats (Mean \pm SEM, $\left.{ }^{*} P<0.05\right)$.

which chronically could lead to left atrial enlargement and fibrosis.

Previous experimental studies reported enhanced AFinducibility in acute OSA models, likely mediated by imbalances in the autonomic system [22,23]. Iwasaki et al. [24] suggested a role for transient LA distension in AF substrate in an acute OSA model in obese rats. Few experimental works have studied the effects of OSA in cardiac structure at the histological level. Simpson et al. [25] showed that intermittent respiratory occlusions acutely induce multifocal areas of necrosis in both ventricles, and Chen et al. [26] described LV myocyte hypertrophy and apoptosis in a chronic intermittent hypoxia model. Neither of these two works studied atrial remodeling. To the best of our knowledge, our work is the first to describe the development of an arrhythmogenic structural substrate in the atria in a chronic OSA model.

Chamber-specific myocardial fibrosis (selective atrial fibrosis while preserving the ventricles) has been observed in our and other experimental settings [20,27-29]. Various mechanisms explain increased atrial susceptibility to fibrosis. Differential stretch and mechanical-loading properties
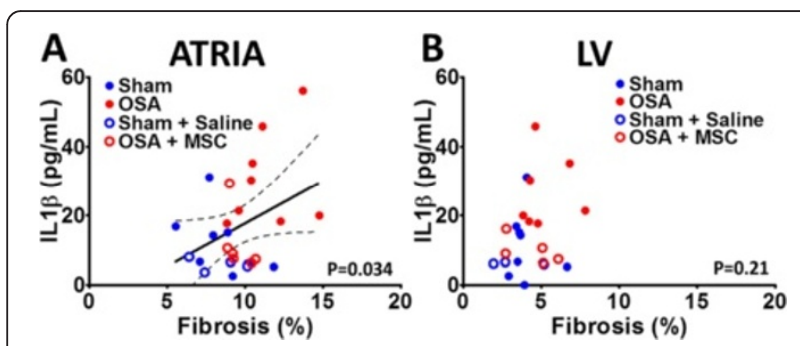

Figure 5 Correlation between plasma IL-1 $\beta$ and myocardial fibrosis at the atrial (A) and ventricular (B) level. Dashed line means $95 \%$ confidence interval. between the atria and ventricles [30], differences in atrialand ventricular-fibroblast reactivity [31], and increased fibrotic response to myocardial ACE activity in the atria [32] have been reported and may contribute to atrial fibrotic susceptibility in our model.

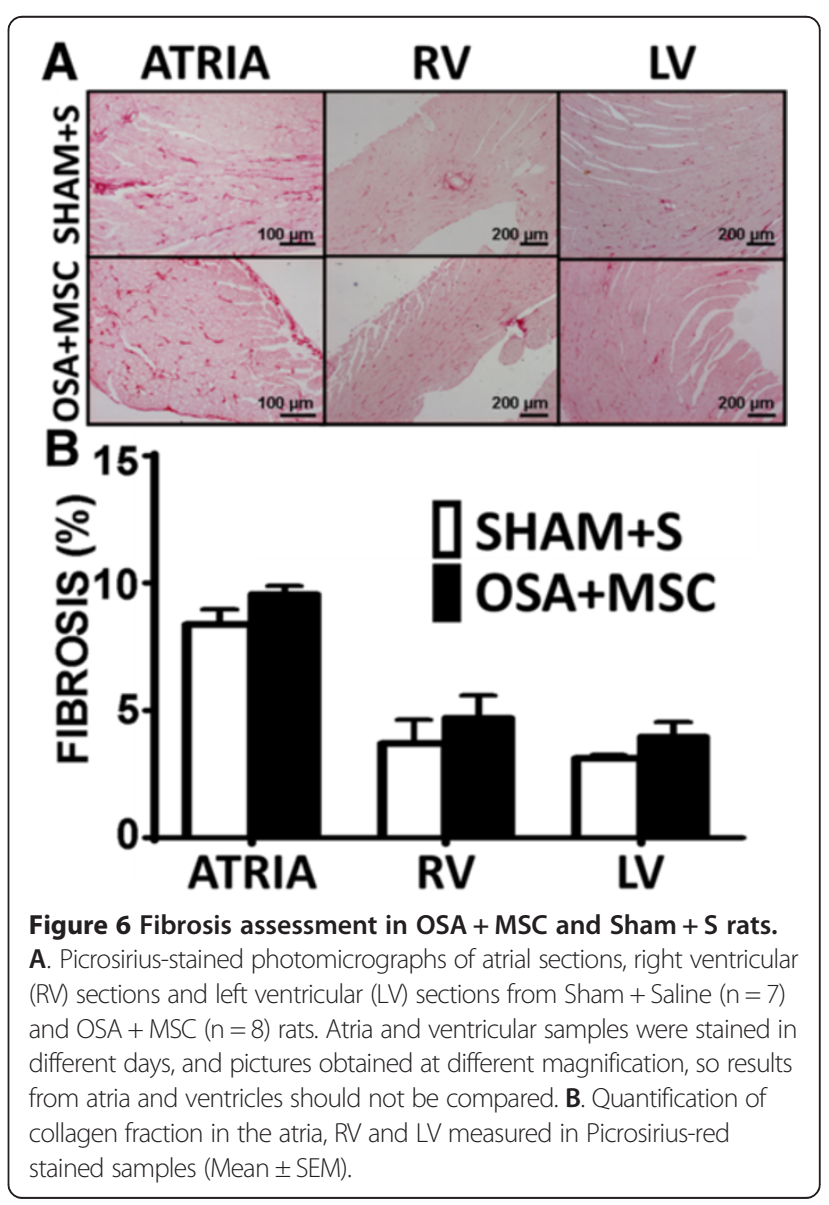




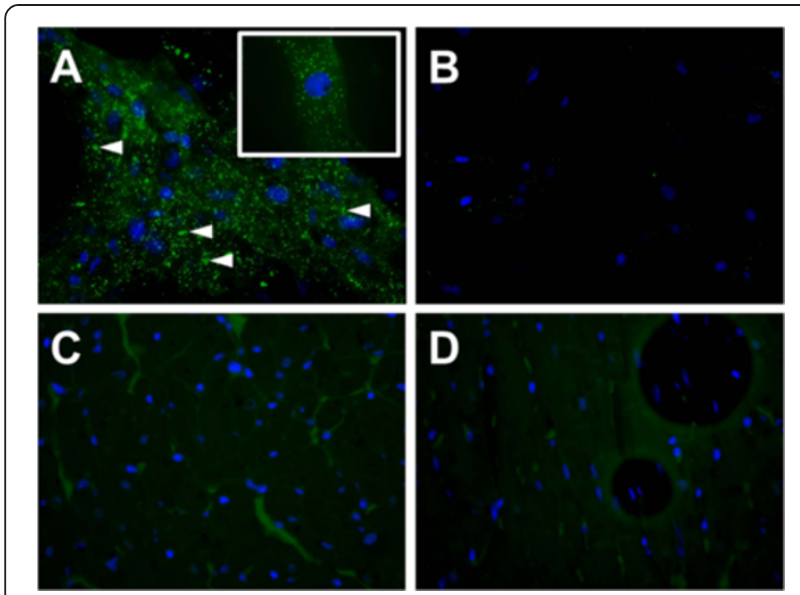

Figure 7 CD90 immunofluorescence myocardial stained samples. A. Cultured MSC, positive control for CD90 (40x). 100x augmentation in the upper box. Abundant CD90 deposits (some marked with arrowhead) are present. B. Cultured MSC, negative control (x40, no anti-CD90 antibody added). C. Sample of interest (left atrium), CD90 stained. No positive cells are marked (x40). D. Sample of interest, negative control ( $x 40$, no anti-CD90 antibody added). Blue: nuclei (DAPI); green: CD90.

The mechanisms by which OSA induces atrial fibrosis are unknown. Our results are summarized in Figure 10 and yield mechanistic insights. Myocardial collagen content is critically dependent on the balance between synthesis and degradation. In the present OSA-model, both ACE upregulation [32] and MMP-2 downregulation [33] could promote myocardial fibrosis. Nevertheless, further data from our work suggest that MMP-2 is central in OSA-induced atrial fibrosis, while the role of ACE is negligible. First, ACE expression was increased in all cardiac chambers in the OSA-group, but increased fibrosis was only found in the atria. Remarkably, MMP-2 was selectively downregulated in the atria. Second, TGF- $\beta 1$, an important fibrotic mediator and an ACE downstream mediator, was not increased in OSA-rats. Results after infusion of MSC further emphasize a critical role for MMP2. OSA-rats in the MSC-treated group had an atrial collagen fraction similar to the Sham $+\mathrm{S}$ group. This finding was accompanied by a normalization in MMP-2 synthesis, likely leading to a higher extracellular matrix degradation activity and hence, reduced fibrosis. Notably, although MSC infusion prevented atrial fibrosis, it was not able to prevent OSA-induced ACEincrease, thus suggesting that increased ACE expression by itself is not enough to sustain OSA-induced atrial fibrosis.

The expression of the remaining collagen degradation (MMP-3, MMP-9, MMP-10, TIMP-1, TIMP-2) and collagen cross-linking (LOX) mediators were not affected in our model.
Besides a profibrotic effect, a growing core of evidence points to OSA as a pro-inflammatory disease [34]. Previous work by our group showed that OSA induces a systemic inflammatory response in an acute animal model. This response was characterized by a significant increase in IL-1 $\beta$ plasmatic levels that was prevented by MSC infusion [11]. The results of the present work confirm this finding in a chronic OSA model and show an increase in the pro-inflammatory cytokine IL-6 in both atria and the LV. Inflammation and fibrosis are closely linked, with several interrelated metabolic pathways. For example, IL-6 is involved in the development of myocardial fibrosis in other experimental settings [35]. Moreover, plasma IL-1 $\beta$ selectively correlated to atrial fibrosis intensity, further highlighting higher fibroblast reactivity in the atria than in the LV [31]. Interestingly, both systemic (IL-1 $\beta$ ) and local (IL-6) inflammatory responses were prevented by MSC infusion. Notably, a growing core of evidence is suggesting that an anti-inflammatory effect underlies beneficial effects of MSC $[12,36]$.

Bone marrow MSC properties including pluripotency, avoidance of detection by the host immune system, and ease of expansion in culture make them an attractive option for cell therapy $[13,14]$. Several studies have shown that MSC transplantation significantly decreases fibrosis in the heart [16,17], lung [37], kidney [38], skin [39] and liver [40]. Although the mechanisms whereby MSCs reduce tissue fibrosis remain unclear, previous studies support our finding that MSC-induced MMP-upregulation might be a hallmark of their antifibrotic effect. In mice skin, Wu et al. [39] demonstrated an antifibrotic effect of bone marrow MSC that was partially mediated by an increased MMP-2 collagen-degradation. Mias et al. [15] showed that MSC injection reduced ventricular fibrosis in a rat model of myocardial infarction by stimulating MMP-2 and MMP-9 secretion in fibroblasts. Consistent with a central role of MMP-2, MSC collagen-accumulation prevention was lost in MMP-2 knock-out fibroblasts [15]. This effect is probably mediated by secreted factors in a paracrine/endocrine mechanism [15,41]. Our data evidenced lack of MSC engrafted in the myocardium and support a systemic effect of MSC in this rat OSA-model.

The development of atrial fibrosis in this OSA model might have important clinical implications if confirmed in humans. First, it demonstrates that pro-arrhythmogenic remodeling can be directly caused by OSA and not limited to remodeling induced by other comorbidities such as hypertension, obesity, coronary artery disease, or diabetes, all of which are very prevalent in OSA patients [21]. Accordingly, OSA screening might be important in AFpatients in order to establish a cause for the arrhythmia. Second, it may provide relevant prognostic information, as both OSA and atrial fibrosis predict poor outcomes of 


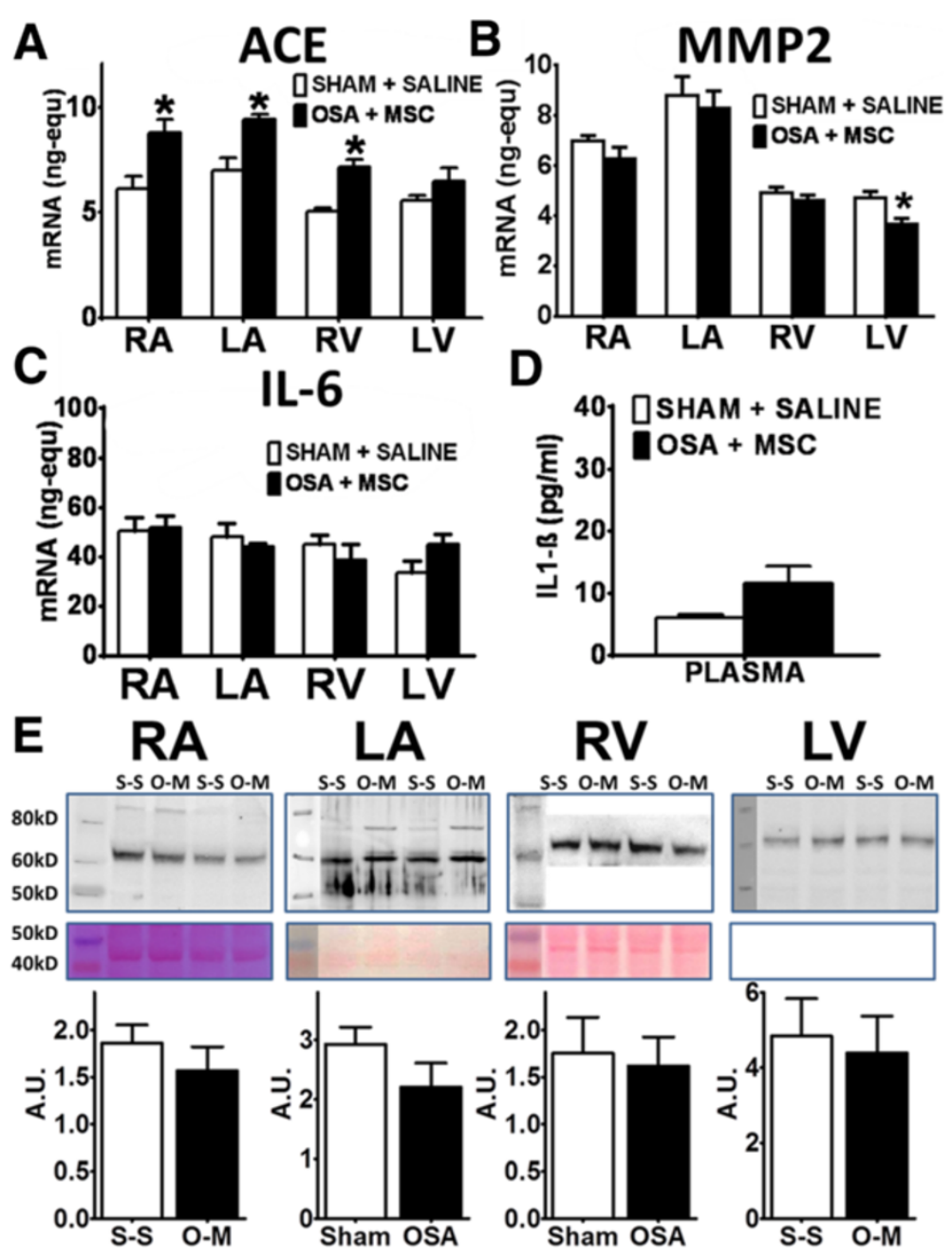

Figure 8 Assessment of fibrosis mechanisms in OSA + MSC vs Sham + S rats. (A) ACE, (B) MMP-2 and (C) IL-6 mRNA expression (ng-equ) in the four cardiac chambers of Sham + Saline $(n=7)$ and OSA + MSC $(n=8)$ rats (Mean \pm SEM $* P<0.05)$. D. Plasmatic concentration $(p g / m L)$ of $I L-1 \beta$ in Sham + Saline and OSA + MSC rats (Mean $\pm S E M * P<0.05)$. E. MMP-2 protein levels quantification. Representative MMP-2 blots (upper panel), Ponceau-stained membranes (middle panel) and normalized quantification (lower panel) (Mean \pm SEM). $n=4-6 / g r o u p$. Left lane is a molecular-weight marker lane; the picture was obtained with visible light. A.U.: Arbitrary Units.
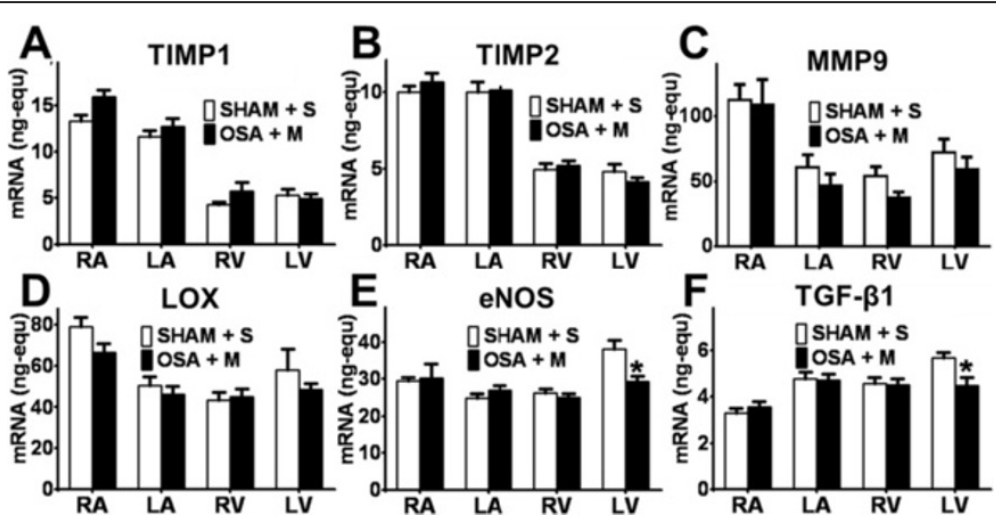

Figure 9 mRNA expression in the OSA + MSC and Sham + S rats. (A) TIMP-1, (B) TIMP-2, (C) MMP-9, (D) LOX, (E) eNOS and (F) TGF- $\beta 1$ mRNA expression (ng-equ) in the four cardiac chambers of Sham + Saline $(n=7)$ and OSA + MSC $(n=8)$ rats (Mean \pm SEM *P $<0.05)$. 


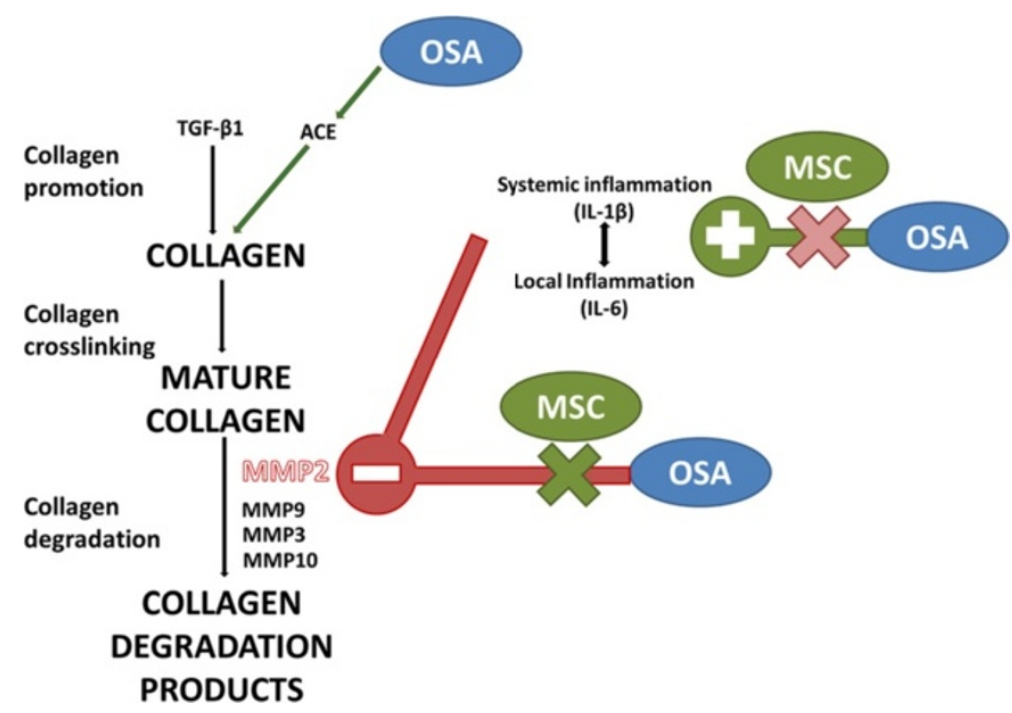

Figure 10 Proposed pathophysiology of OSA-induced atrial fibrosis and MSC mechanism of action. OSA acts as a pro-inflammatory stimulus and inhibits MMP-2 synthesis, reducing collagen degradation and thus favoring collagen accumulation. MSC administration blunts inflammation and normalizes MMP-2 synthesis, thereby increasing collagen degradation and preventing from collagen deposit. OSA also increases ACE expression, but its role in fibrosis promotion is uncertain. Red lines represent inhibition, green lines represent activation. MSC: mesenchymal stem cells. OSA: obstructive sleep apnea.

AF treatments. Finally, as OSA is a treatable disorder, early detection and treatment by means of CPAP or newer therapies may slow AF substrate progression and improve outcomes of antiarrhythmic drugs, electrical cardioversion, or AF ablation. Our results also suggest that MSC might have the potential to prevent the atrial profibrotic remodeling induced by OSA, but its clinical implication in humans needs to be demonstrated.

\section{Limitations}

Some limitations should be acknowledged and taken into consideration when interpreting our results. First, we lack of electrophysiological and AF inducibility studies and thus, increased inducibility cannot be ensured from our results. However, we show that OSA induces atrial fibrosis, a hallmark of AF and a well-known AF promoter [8], at similar intensity to other reports showing increased inducibility [42]. Second, we assessed protein levels for some genes, including collagen deposit, inflammatory cytokines and MMP-2, but failed to obtain blots for ACE. Third, we only assessed atrial fibrosis as AF substrate; additional studies focusing on atrial dilation or electrical remodeling are warranted.

\section{Conclusions}

In conclusion, our model demonstrates that OSA induces the development of atrial fibrosis, an arrhythmogenic substrate that might explain the association between OSA and atrial fibrillation. Fibrosis might be mediated by increased atrial expression of IL- 6 and decreased atrial expression of MMP-2 with the subsequent decline in collagen-degradation. This atrial fibrosis is prevented by the intravenous administration of MSC, which normalizes IL-6 and MMP-2 expression.

\section{Competing interests}

The authors declare that they have no competing interests.

\section{Authors' contributions}

LM, IA, JMM, JB, MB and PR: Conceptualized and designed the study. RF, JMM, MT, and IA: Developed and carried out OSA murine model. PR, CR and MB: Performed histological, plasma, mRNA and protein analysis and data interpretation. PR: Performed statistical analysis and wrote the first draft. All authors read and approved the final manuscript.

\section{Acknowledgments}

The authors want to thank Neus Portella for editing assistance and Nadia Castillo for her excellent technical help.

\section{Funding sources}

This work was supported by a grant (PI10/00834) from the Fondo de Investigación Sanitaria and cofinanced by the ISCIII- Subdirección General de Evaluación and the European Regional Development Fund (FEDER); Ministerio de Economía y Competitividad (SAF2011-22576); REDINSCOR [V-2006-RET0308-O] and a grant from Hospital Clínic, Barcelona, Catalonia, Spain (Premi Emili Letang to PR).

\section{Author details}

Thorax Institute, Unitat de Fibril · lació Auricular, Hospital Clínic. Universitat de Barcelona and Institut d'Investigacions Biomèdiques August Pi i Sunyer (IDIBAPS), Barcelona, Catalonia, Spain. ²Unitat del Son. Servei Pneumologia, Hospital Clínic. Universitat de Barcelona IDIBAPS-CIBERES Barcelona, Barcelona, Catalonia, Spain. ${ }^{3}$ Unitat de Biofísica i Bioenginyeria, Facultat de Medicina, Universitat de Barcelona-IDIBAPS-CIBERES, Barcelona, Catalonia, Spain. 
Received: 16 October 2013 Accepted: 18 March 2014

Published: 28 April 2014

\section{References}

1. Gami AS, Pressman G, Caples SM, Kanagala R, Gard JJ, Davison DE, Malouf JF, Ammash NM, Friedman PA, Somers VK: Association of atrial fibrillation and obstructive sleep apnea. Circulation 2004, 110:364-367.

2. Gami AS, Hodge DO, Herges RM, Olson EJ, Nykodym J, Kara T, Somers VK: Obstructive sleep apnea, obesity, and the risk of incident atrial fibrillation. J Am Coll Cardiol 2007, 49:565-571.

3. Kanagala R, Murali NS, Friedman PA, Ammash NM, Gersh BJ, Ballman KV, Shamsuzzaman AS, Somers VK: Obstructive sleep apnea and the recurrence of atrial fibrillation. Circulation 2003, 107:2589-2594.

4. Matiello M, Nadal M, Tamborero D, Berruezo A, Montserrat J, Embid C, Rios J, Villacastin J, Brugada J, Mont L: Low efficacy of atrial fibrillation ablation in severe obstructive sleep apnoea patients. Europace 2010, 12:1084-1089.

5. Ng CY, Liu T, Shehata M, Stevens S, Chugh SS, Wang X: Meta-analysis of obstructive sleep apnea as predictor of atrial fibrillation recurrence after catheter ablation. Am J Cardiol 2011, 108:47-51.

6. Monahan K, Brewster J, Wang L, Parvez B, Goyal S, Roden DM, Darbar D: Relation of the severity of obstructive sleep apnea in response to anti-arrhythmic drugs in patients with atrial fibrillation or atrial flutter. Am J Cardiol 2012, 110:369-372.

7. Latina JM, Estes NA 3rd, Garlitski AC: The Relationship between Obstructive Sleep Apnea and Atrial Fibrillation: A Complex Interplay. Pulm Med 2013, 2013:621736.

8. Burstein B, Nattel S: Atrial fibrosis: mechanisms and clinical relevance in atrial fibrillation. J Am Coll Cardiol 2008, 51:802-809.

9. Mahnkopf C, Badger TJ, Burgon NS, Daccarett M, Haslam TS, Badger CT, McGann CJ, Akoum N, Kholmovski E, Macleod RS, Marrouche NF: Evaluation of the left atrial substrate in patients with lone atrial fibrillation using delayed-enhanced MRI: implications for disease progression and response to catheter ablation. Heart Rhythm 2010, 7:1475-1481.

10. Almendros I, Farre R, Torres M, Bonsignore MR, Dalmases M, Ramirez J, Navajas D, Montserrat JM: Early and mid-term effects of obstructive apneas in myocardial injury and inflammation. Sleep Med 2011, 12:1037-1040

11. Carreras A, Almendros I, Montserrat JM, Navajas D, Farre R: Mesenchymal stem cells reduce inflammation in a rat model of obstructive sleep apnea. Respir Physiol Neurobiol 2010, 172:210-212.

12. Iyer S, Rojas M: Anti-inflammatory effects of mesenchymal stem cells: novel concept for future therapies. Expert Opin Biol Ther 2008, 8:569-581.

13. Pittenger MF, Martin BJ: Mesenchymal stem cells and their potential as cardiac therapeutics. Circ Res 2004, 95:9-20.

14. Jiang $Y$, Jahagirdar $B N$, Reinhardt RL, Schwartz RE, Keene CD, OrtizGonzalez XR, Reyes M, Lenvik T, Lund T, Blackstad M, Du J, Aldrich S, Lisberg A, Low WC, Largaespada DA, Verfaillie CM: Pluripotency of mesenchymal stem cells derived from adult marrow. Nature 2002, 418:41-49.

15. Mias C, Lairez O, Trouche E, Roncalli J, Calise D, Seguelas M, Ordener C, Piercecchi-Marti M, Auge N, Salvayre A, Bourin P, Parini A, Cussac D: Mesenchymal Stem Cells Promote Matrix Metalloproteinase Secretion by Cardiac Fibroblasts and Reduce Cardiac Ventricular Fibrosis After Myocardial Infarction. Stem Cells 2009, 27:2734-2743.

16. Nagaya $N$, Kangawa $K$, Itoh $T$, Iwase $T$, Murakami S, Miyahara $Y$, Fujii $T$, Uematsu M, Ohgushi H, Yamagishi M, Tokudome T, Mori H, Miyatake K, Kitamura S: Transplantation of mesenchymal stem cells improves cardiac function in a rat model of dilated cardiomyopathy. Circulation 2005, 112:1128-1135.

17. Li L, Zhang Y, Li Y, Yu B, Xu Y, Zhao S, Guan Z: Mesenchymal stem cell transplantation attenuates cardiac fibrosis associated with isoproterenol-induced global heart failure. Transpl Int 2008, 21:1181-1189.

18. Almendros I, Carreras A, Montserrat JM, Gozal D, Navajas D, Farre R: Potential role of adult stem cells in obstructive sleep apnea. Front Neurol 2012, 3:112.

19. Farre R, Nacher M, Serrano-Mollar A, Galdiz JB, Alvarez FJ, Navajas D, Montserrat JM: Rat model of chronic recurrent airway obstructions to study the sleep apnea syndrome. SLEEP 2007, 30:930-933.
20. Benito B, Gay-Jordi G, Serrano-Mollar A, Guasch E, Shi Y, Tardif JC, Brugada J, Nattel S, Mont L: Cardiac arrhythmogenic remodeling in a rat model of long-term intensive exercise training. Circulation 2011, 123:13-22.

21. Kasai T, Floras JS, Bradley TD: Sleep Apnea and Cardiovascular Disease: A Bidirectional Relationship. Circulation 2012, 126:1495-1510.

22. Ghias M, Scherlag BJ, Lu Z, Niu G, Moers A, Jackman WM, Lazzara R, Po SS: The role of ganglionated plexi in apnea-related atrial fibrillation. J Am Coll Cardiol 2075-2083, 2009:54.

23. Linz D, Schotten U, Neuberger HR, Bohm M, Wirth K: Negative tracheal pressure during obstructive respiratory events promotes atrial fibrillation by vagal activation. Heart Rhythm 2011, 8:1436-1443.

24. Iwasaki YK, Shi Y, Benito B, Gillis MA, Mizuno K, Tardif JC, Nattel S: Determinants of atrial fibrillation in an animal model of obesity and acute obstructive sleep apnea. Heart Rhythm 2012, 9:1409-1416. e1401.

25. Simpson JA, Brunt KR, Iscoe S: Repeated inspiratory occlusions acutely impair myocardial function in rats. $J$ Physiol 2008, 586:2345-2355.

26. Chen L, Zhang J, Gan T, Chen-Izu Y, Hasday J, Karmazyn M, Balke C, Scharf S: Left ventricular dysfunction and associated cellular injury in rats exposed to chronic intermittent hypoxia. J Appl Physiol 2008, 104:218-223.

27. Li D, Fareh S, Leung TK, Nattel S: Promotion of Atrial Fibrillation by Heart Failure in Dogs : Atrial Remodeling of a Different Sort. Circulation 1999, 100:87-95.

28. Hanna N, Cardin S, Leung TK, Nattel S: Differences in atrial versus ventricular remodeling in dogs with ventricular tachypacing-induced congestive heart failure. Cardiovasc Res 2004, 63:236-244.

29. Nakajima H, Nakajima HO, Salcher O, Dittie AS, Dembowsky K, Jing S, Field L: Atrial but Not Ventricular Fibrosis in Mice Expressing a Mutant Transforming Growth Factor- 1 Transgene in the Heart. Circ Res 2000, 86:571-579.

30. Yue L, Xie J, Nattel S: Molecular determinants of cardiac fibroblast electrical function and therapeutic implications for atrial fibrillation. Cardiovasc Res 2011, 89:744-753.

31. Burstein B, Libby E, Calderone A, Nattel S: Differential behaviors of atrial versus ventricular fibroblasts: a potential role for platelet-derived growth factor in atrial-ventricular remodeling differences. Circulation 2008, 117:1630-1641.

32. Xiao H, Fuchs S, Campbell D, Lewis W, Dudley S, Kasi V, Hoit B, Keshelava G, Zhao H, Capecchi M, Bernstein K: Mice with cardiac-restricted angiotensinconverting enzyme (ACE) have atrial enlargement, cardiac arrhythmia, and sudden death. Am J Pathol 2004, 165:1019-1032.

33. Van Linthout S, Seeland U, Riad A, Eckhardt O, Hohl M, Dhayat N, Richter U, Fischer JW, Bohm M, Pauschinger M, Schultheiss HP, Tschope C: Reduced MMP-2 activity contributes to cardiac fibrosis in experimental diabetic cardiomyopathy. Basic Res Cardiol 2008, 103:319-327.

34. Garvey JF, Taylor CT, McNicholas WT: Cardiovascular disease in obstructive sleep apnoea syndrome: the role of intermittent hypoxia and inflammation. Eur Respir J 2009, 33:1195-1205.

35. Melendez GC, McLarty JL, Levick SP, Du Y, Janicki JS, Brower GL: Interleukin 6 mediates myocardial fibrosis, concentric hypertrophy, and diastolic dysfunction in rats. Hypertension 2010, 56:225-231.

36. van den Akker F, Deddens JC, Doevendans PA, Sluijter JP: Cardiac stem cell therapy to modulate inflammation upon myocardial infarction. Biochim Biophys Acta 1830, 2013:2449-2458

37. Ortiz LA, Gambelli F, McBride C, Gaupp D, Baddoo M, Kaminski N, Phinney DG: Mesenchymal stem cell engraftment in lung is enhanced in response to bleomycin exposure and ameliorates its fibrotic effects. Proc Natl Acad Sci U S A 2003, 100:8407-8411.

38. Ninichuk V, Gross O, Segerer S, Hoffmann R, Radomska E, Buchstaller A, Huss R, Akis N, Schlondorff D, Anders HJ: Multipotent mesenchymal stem cells reduce interstitial fibrosis but do not delay progression of chronic kidney disease in collagen4A3-deficient mice. Kidney Int 2006, 70:121-129.

39. Wu Y, Huang S, Enhe J, Ma K, Yang S, Sun T, Fu X: Bone marrow-derived mesenchymal stem cell attenuates skin fibrosis development in mice. Int Wound J, Published online on 2013 February 5. doi:10.1111/iwj.12034.

40. Iwamoto T, Terai S, Hisanaga T, Takami T, Yamamoto N, Watanabe $\mathrm{S}$, Sakaida I: Bone-marrow-derived cells cultured in serum-free medium reduce liver fibrosis and improve liver function in carbon-tetrachloridetreated cirrhotic mice. Cell Tissue Res 2013, 351:487-495.

41. Mao Q, Lin CX, Liang XL, Gao JS, Xu B: Mesenchymal stem cells overexpressing integrin-linked kinase attenuate cardiac fibroblast 
proliferation and collagen synthesis through paracrine actions. Mol Med Rep 2013, 7:1617-1623.

42. Guasch E, Benito B, Qi X, Cifelli C, Naud P, Shi Y, Mighiu A, Tardif JC, Tadevosyan A, Chen Y, Gillis MA, Iwasaki YK, Dobrev D, Mont L, Heximer S,

Nattel S: Atrial fibrillation promotion by endurance exercise:

demonstration and mechanistic exploration in an animal model. J Am Coll Cardiol 2013, 62:68-77.

doi:10.1186/1465-9921-15-54

Cite this article as: Ramos et al: Atrial fibrosis in a chronic murine model of obstructive sleep apnea: mechanisms and prevention by mesenchymal stem cells. Respiratory Research 2014 15:54.

\section{Submit your next manuscript to BioMed Central and take full advantage of:}

- Convenient online submission

- Thorough peer review

- No space constraints or color figure charges

- Immediate publication on acceptance

- Inclusion in PubMed, CAS, Scopus and Google Scholar

- Research which is freely available for redistribution 